\title{
ILCEA
}

Revue de l'Institut des langues et cultures

d'Europe, Amérique, Afrique, Asie et Australie

24 | 2015

Lire et écrire ensemble

\section{Éditer et lire Borges en France (1939-1970). Pour une poétique de la réception}

Editing and Reading Borges in France (1939-1970). A Reception Theory

Editar y leer a Borges en Francia (1939-1970). Para una poética de la recepción

\section{Michel Lafon}

\section{CpenEdition}

\section{Journals}

Édition électronique

URL : http://journals.openedition.org/ilcea/3659

DOI : 10.4000/ilcea.3659

ISSN : 2101-0609

Éditeur

UGA Éditions/Université Grenoble Alpes

Édition imprimée

ISBN : 978-2-84310-313-1

ISSN : 1639-6073

Référence électronique

Michel Lafon, «Éditer et lire Borges en France (1939-1970). Pour une poétique de la réception », ILCEA

[En ligne], 24 | 2015, mis en ligne le 16 octobre 2015, consulté le 01 mai 2019. URL : http://

journals.openedition.org/ilcea/3659 ; DOI : 10.4000/ilcea.3659

Ce document a été généré automatiquement le 1 mai 2019.

(c) ILCEA 


\title{
Éditer et lire Borges en France (1939-1970). Pour une poétique de la réception
}

\author{
Editing and Reading Borges in France (1939-1970). A Reception Theory \\ Editar y leer a Borges en Francia (1939-1970). Para una poética de la recepción
}

Michel Lafon

\section{Jorge Luis Borges, la Poétique, la littérature argentine}

1 J'ai commencé à lire l'œuvre de Jorge Luis Borges en espagnol à l'âge de dix-sept ans, en 1971, par hasard, pour améliorer mon niveau en espagnol pendant l'été précédant mon entrée en classes préparatoires littéraires, à Montpellier. J'ai aussitôt senti que cette lecture (les nouvelles des recueils Ficciones et El Aleph) allait jouer un rôle capital dans ma vie. Un an plus tard, à mon entrée en deuxième année de classes préparatoires, si j'ai opté pour la spécialité « espagnol » de la préparation au concours, ce fut en grande partie sous l'influence de cette découverte. L'année suivante, reçu au concours d'entrée à l'École normale supérieure (ENS) de Saint-Cloud et m'inscrivant parallèlement en licence d'espagnol à la Sorbonne, je retrouvais Ficciones de Borges au programme de troisième année de l'Institut d'études hispaniques de la rue Gay-Lussac. C'est dire que, bien que mon entrée dans l'hispanisme ait été précoce, j'ai été " borgiste » avant même de devenir hispaniste. Depuis, je n'ai plus cessé de l'être: une première thèse (thèse de troisième cycle), de 1978 à 1981, consacrée à l'œuvre poétique de Borges, une seconde (thèse d'État), de 1981 à 1989, consacrée à l'ensemble du corpus borgésien (nouvelles, essais et poèmes), les deux sous la direction de mon maitre Maurice Molho, professeur à la Sorbonne, ont été accompagnées ou suivies de publications innombrables, qui ne se sont pas interrompues jusqu'à ce jour.

2 Ma scolarité à l'ENS de Saint-Cloud (1973-1977), qui s'est achevée par l'obtention de l'agrégation d'espagnol, a constitué l'étape clé de ma formation intellectuelle et 
scientifique : c'est pendant ces années que j'ai découvert la Poétique, et plus précisément la Nouvelle Critique, grâce aux ouvrages de Roland Barthes, Gérard Genette, Jean Ricardou et quelques autres, qui sont devenus mes livres de chevet. Jusque-là, ma scolarité avait été marquée, au lycée par un double cursus à la fois classique (françaislatin-grec) et scientifique (mathématiques-physique), puis en classes préparatoires par deux années généralistes très denses, selon la tradition, où pour la première fois j'entrais véritablement dans la profondeur de certains corpus littéraires, essentiellement français. Mais c'est donc à dix-neuf ans que j'ai eu la révélation que la littérature et le texte pouvaient être les enjeux les plus savoureux du savoir et de la réflexion, autrement dit que ma passion littéraire et poéticienne naissante pouvait orienter la suite de ma vie. Surtout, j'ai constaté avec stupéfaction que beaucoup des choses que je lisais, chez Barthes, chez Genette, chez Ricardou et chez d'autres, Borges les avait déjà dites, à sa façon bien sûr, dès les années 1930 ou 1940, aussi bien dans ses nouvelles que dans ses essais. Pour n'en donner qu'un exemple : lorsque, à la fin de «Pierre Ménard, auteur du Quichotte » (1939), le narrateur de la nouvelle écrit : «Ménard (peut-être sans le vouloir) a enrichi l'art figé et rudimentaire de la lecture par une technique nouvelle : la technique de l'anachronisme délibéré et des attributions erronées ", ce n'est pas seulement, à mes yeux, la Nouvelle Critique qu'il « programme » avec plus de vingt ans d'avance, c'est aussi ce que je me plais à appeler la « Nouvelle Nouvelle Critique », dont je situe l'émergence au tournant des années 1970-1980, avec des ouvrages comme La Seconde Main ou le travail de la citation, d'Antoine Compagnon (1979), et Palimpsestes ou la littérature au second degré, de Gérard Genette (1982) : ce n'est plus le texte en soi qui est l'objet privilégié de la théorie littéraire, c'est le jeu des textes entre eux qui passe alors au cœur des interrogations poéticiennes, tel que Borges le faisait miroiter dans ce final fulgurant et ludique, qu'on l'appelle citation (Compagnon) ou transtextualité (Genette). C'est de cette « Nouvelle Nouvelle Critique » que je me réclame, ayant vite trouvé encore plus de charme à l'étude des relations transtextuelles - et notamment hypertextuelles - qu'à l'analyse narratologique de tel ou tel texte précis. Et c'est sous cette bannière que s'inscrit ma première publication majeure, Borges ou la réécriture, livre paru en 1990 au Seuil dans la collection « Poétique ", où je retrouvais ainsi les théoriciens qui m'avaient nourri.

Sans le savoir, je me suis trouvé placé dans une situation privilégiée : j'ai été sans doute le premier à lire Borges dans sa langue originale à la lumière d'une formation en Poétique (et réciproquement : le premier à lire la Nouvelle Critique sous l'inspiration du Borges original). Les chercheurs latino-américains qui lisent Borges dans les années 1950-1980 optent généralement pour de féroces leçons idéologiques ou pour un thématisme assez réducteur et, quand ils s'occupent de textualité (à de notables exceptions près, telle la figure tutélaire de Noé Jitrik, dans El fuego de la especie, 1971), ils le font à partir d'une vague imprégnation théorique, issue de textes de seconde main (si je puis dire). Quant aux théoriciens français, à part un Genette, qui peut lire Borges dans l'original (et sans parler d'un Paul Bénichou, qui a été proche de Borges et connaît parfaitement l'espagnol, mais qui ne se réclame pas de la Nouvelle Critique), la quasi-totalité se contente de le lire en français, ce qui n'est pas sans conséquence, j'y reviendrai - puisque c'est tout le problème. Me situer à ce carrefour linguistique et théorique m'a permis d'écrire ce premier livre (prolongement de ma thèse d'État), et n'a cessé depuis d'inspirer ma recherche, en une sorte de va-et-vient permanent et stimulant entre lecture de Borges et théorisation littéraire, de constante relance de l'une par l'autre. 
4 J'ai été élu maitre-assistant (1983) puis professeur (1991) à l'université Stendhal de Grenoble. À l'occasion de ma délégation junior à l'IUF (1993-1998), j'y ai fondé une "chaire de littérature argentine» unique en France et dans le monde (hors de l'Argentine), et j'ai finalement créé une école argentiniste qui est elle aussi, sans doute, unique en France, qui a donné à ce jour un professeur et une dizaine de maîtres de conférences argentinistes aux universités françaises, et qui est reconnue internationalement. Je tiens un séminaire mensuel, qui est l'occasion pour les participants (étudiants avancés, doctorants, collègues grenoblois ou non grenoblois) de mener une recherche collective sur un sujet théorique appliqué aux littératures du Río de la Plata, dont les résultats sont ensuite publiés dans notre revue Tigre: à titre indicatif, ces dernières années, nous avons travaillé sur les formes littéraires brèves et hyperbrèves, sur la science-fiction et sur le romanesque. Dans le prolongement de mon séminaire, j'ai œuvré à la mise en place d'un réseau argentiniste inter-universitaire, avec des collègues des universités de Caen, de Lille 3, de Lyon 2, de Paris 4, de Paris 8, etc. - en relation constante avec des collègues argentins (université de Buenos Aires, université catholique argentine, université nationale de Rosario...). Pour ne citer que deux réalisations: les universités de Paris 8 et de Grenoble 3 ont organisé (à Paris et à Grenoble) un grand colloque international consacré à l'œuvre de l'écrivain argentin César Aira, en 2004; et en 2008, les universités de Caen, de Paris 4, de Paris 8 et de Grenoble 3 ont organisé (à la Sorbonne) un colloque international sur l'œuvre d'un autre écrivain argentin majeur, Ricardo Piglia, qui avait été précédé d'une journée d'études à Grenoble à l'automne 2007, toujours en présence de l'auteur.

5 C'est dire que mon intérêt pour la littérature argentine ne se limite évidemment pas à Borges. Progressivement, je me suis ouvert à l'ensemble de la littérature de ce pays. Un saut quantitatif et qualitatif majeur a été accompli en 1995, lorsque ma délégation « junior » à l'IUF m'a permis de réaliser un premier séjour à Buenos Aires (neuf autres ont suivi à ce jour). En quelques semaines, j'ai rencontré la quasi-totalité des principaux écrivains actuels (ainsi que des universitaires, des éditeurs, des artistes...), ai noué avec eux des liens très forts et, lisant désormais leur production au fur et mesure de sa parution, je suis enfin devenu un argentiniste à part entière, qui avait déjà une bonne connaissance de la littérature du xix siècle et de celle des deux premiers tiers du $\mathrm{xx}^{\mathrm{e}}$, mais à qui il manquait jusque-là la connaissance immédiate et systématique de la production fictionnelle d'aujourd'hui.

Un mot particulier sur César Aira, sans doute l'écrivain argentin vivant le plus important : choqué de voir qu'il était quasiment inconnu en France, alors que ses livres que je lisais depuis le tout début des années 1990 m'émerveillaient, je me suis donné pour mission de le faire connaitre en France : ce rôle de passeur de la littérature argentine m'a semblé capital pour un universitaire, et j'ai finalement traduit onze de ses romans, qui constituent le cœur de son œuvre. Ce sont sans doute ces rôles multiples de spécialiste, d'enseignant, de chercheur, de directeur de recherche, de pilote d'un réseau national et international d'argentinistes, de traducteur, de passeur et finalement d'amoureux de leur culture et tout particulièrement de leur littérature que les membres de l'Académie argentine des Lettres ont voulu saluer en m'élisant à l'unanimité, en mai 2010, membre correspondant de leur Académie pour la France. 


\section{Pour une poétique de la réception}

7 Ce n'est qu'au milieu des années 1980 que j'ai commencé à lire Borges en français, essentiellement parce que j'ai ouvert à cette époque un séminaire de traductologie littéraire à l'université Stendhal, lorsque j'y ai fondé le diplôme d'études approfondies d'espagnol ( $5^{\mathrm{e}}$ année d'études), et que l'œuvre borgésienne en est devenue naturellement le corpus de référence: l'œuvre poétique, notamment, me permettait de proposer aux étudiants des textes connaissant plusieurs versions en espagnol - Borges n'ayant cessé de les réécrire des années 1920 jusqu'aux années 1960 -, ainsi que des traductions en français, en italien, en anglais, etc. - avec souvent deux ou plusieurs traductions dans une même langue, dues à des traducteurs successifs. La simple observation de la manière dont l'œuvre poétique de Borges est traduite en français fait d'ailleurs apparaître de telles aberrations que l'on peut dire que cette œuvre poétique n'existe pas en français, à l'exception de quelques réussites tardives dues à des traducteurs plus rigoureux. Globalement, un francophone qui ignore l'espagnol ne peut en avoir qu'une très lointaine perception, une image partielle et déformée.

8 C'est sans doute à cette occasion que j'ai pris la pleine conscience de ce qui est pourtant une évidence : un écrivain n'existe pas de toute éternité, dans une espèce d'immanence qui le mettrait directement à la disposition des lecteurs. À l'âge moderne, pas de réception sans édition préalable. Genette a montré exhaustivement dans Seuils (1987) toutes les manières dont le péritexte éditorial induit la lecture d'un livre, tous les effets de lecture qui en découlent. Mon propos est de faire un pas de plus dans la recherche de ces effets de lecture, en me concentrant sur le cas d'un écrivain étranger édité en France : aux filtres initiaux de l'édition originale s'ajoutent ou se substituent les filtres de l'édition dans la nouvelle langue. Je souhaite repérer les effets de sens produits par l'édition au sens large (édition, traduction, diffusion) d'une œuvre littéraire étrangère sur sa lecture au sens large (lecture, critique, interprétation) dans le pays d'arrivée, et les comparer aux effets de sens produits par l'édition d'origine, en quête d'éventuelles distorsions, ou du moins de divergences significatives. Il ne s'agit pas de schématiser les effets de sens produits par l'édition argentine, il ne saurait exister une vérité originelle pure et unique liée à l'édition initiale, voire consubstantielle à l'œuvre avant même qu'elle soit éditée : voilà pourquoi seules des différences nettes pourront être utiles à mon propos. Je propose donc une poétique de la réception de l'œuvre borgésienne en France, sur une période relativement courte et sans doute décisive, allant de la toute première nouvelle traduite en français, en 1939, jusqu'à l'année 1970, où paraissent au Seuil, Evaristo Carriego (essai de 1930) et, chez Gallimard (éditeur principal de Borges en France), CEuvre poétique 1925-1965.

Ces dernières années, commençant à m'intéresser plus systématiquement aux conditions de l'édition de Borges en France, je suis allé de surprise en surprise, qui à ce stade semblent converger: l'éditeur historique de Borges en France, Roger Caillois, dont la légende raconte qu'il créa aux éditions Gallimard la collection «La Croix du Sud » pour faire connaître l'œuvre de Borges (Fictions en constitua de fait le premier volume, en 1951), et dont Borges a souvent dit qu'il lui doit de l'avoir « inventé », entretenait des relations très ambiguës avec Borges (en raison de querelles intellectuelles indissociables de conflits personnels). Le premier traducteur en français, un Argentin ami d'adolescence de Borges, Néstor Ibarra, fait preuve à l'égard de son œuvre de légèreté : l'étude de la toute première fiction borgésienne parue sous sa plume dans la revue Mesures, en 
avril 1939, où tout change par rapport au texte original (le titre, les dates de l'aventure, certains événements ou personnages, beaucoup de références bibliographiques...), et qui est précédée d'une brève notice biographique particulièrement perfide, suffirait à le montrer. Et ainsi, pourrait-on dire, ad nauseam: livres édités dans le désordre, dont le titre et la composition changent en changeant de langue, livres carrément inventés (recomposés par l'éditeur selon un schéma personnel), préfaces ou postfaces hostiles, aveugles à ce qui fait la qualité et la spécificité mêmes de l'œuvre dont elles prétendent pourtant éclairer la compréhension et susciter la promotion, reconduites à l'envi de rééditions en rééditions, changement aléatoire de traducteurs, changement tout aussi aléatoire d'éditeurs, cloisonnement rigide entre les genres même quand Borges se plaît à les transcender, données biographiques et bibliographiques fallacieuses, etc. D'emblée, donc, le sentiment d'une étrangeté généralisée, et celui d'un investissement (esthétique, psychologique... ?) excessif, en tout cas tout à fait hors norme, de l'éditeur principal de la période considérée, et du premier traducteur. Il va de soi que je devrai aussi me pencher sur la façon dont Borges, qui connaît parfaitement le français, réagit ou ne réagit pas aux cahots de sa trajectoire éditoriale française.

10 Je n'irai pas plus loin ici dans cette voie. Ce n'est pas le roman des relations dangereuses ou ambiguës, des amitiés déçues ou trahies qui m'intéresse le plus, même si ce roman est savoureux et mérite sans doute qu'on lui consacre un minimum d'attention. Mais plus que les causes, ce sont donc les effets qui m'occupent. Mon interrogation scientifique principale portera sur les effets éventuels de telles attitudes, de telles options, de tels processus sur la lecture de Borges en France : dans quelle mesure cette édition influe-t-elle sur cette réception, dans quelle mesure la programme-t-elle? Ces effets font-ils un système, construisent-ils un "Borges français" clairement opposable à un "Borges argentin"? Comme toujours avec Borges, je compte sur la dimension excessive de son œuvre pour voir apparaitre des traits que d'autres corpus ne me livreraient sans doute pas, ou bien plus discrètement.

11 J'ai déjà quelques motifs pour estimer que ces effets sont nombreux et puissants. En même temps que je découvrais la manière dont Borges avait été traduit et édité en France, je découvrais en effet la manière dont il était lu. Je ne parle pas ici des comptes rendus ni des critiques, auxquels je reviendrai, mais d'abord de la réception des lecteurs cultivés. J'ai eu à la même époque (milieu des années 1980), à plusieurs reprises, l'occasion d'intervenir devant des publics d'universitaires non hispanophones, et j'ai à chaque fois été frappé par ce qui m'apparaissait comme des a priori de lectures très forts, aux antipodes de ma propre lecture : je citerai pêle-mêle, pour illustrer quelques-uns de ces a priori, le côté sérieux ou même tragique de Borges (alors que son humour éclate à chacune de ses pages, en espagnol), sa dimension réactionnaire, voire fasciste ou antisémite (alors que beaucoup de ses textes, dès les années trente, dénonçant la montée du nazisme, s'inventant une "judaïté d'honneur», condamnant les turpitudes de ses compatriotes fascinés par Hitler, sont d'une rare et admirable lucidité), une certaine ironie même vis-à-vis de ses jeux narratifs (alors qu'ils constituent sans doute une des inventions littéraires majeures du vingtième siècle)... Bref, le «Borges » que lisaient en français mes compatriotes n'était pas celui que j'avais lu en argentin: je ne peux m'empêcher de faire le lien entre ces lectures « décalées », « déplacées », et les bizarreries accompagnant sa diffusion en France.

12 À ce stade de mon projet, je me dois d'éviter les leçons prématurées, comme tirées une fois pour toutes et que l'étude à venir se contenterait de confirmer. Par exemple, on ne 
peut nier que la France a joué un rôle pionnier et décisif pour la diffusion de Borges dans le reste du monde. Il y a là un paradoxe que ma recherche devra intégrer: l'édition française a sans doute agi d'une étrange façon, mais elle a agi; de même que la critique française (j'y reviendrai), avec ses bonheurs et ses malheurs, a sans doute été la première au monde à constituer l'œuvre borgésienne en champ d'interrogation privilégié, et même à l'instaurer en véritable clé de la modernité.

La période choisie est celle où les critiques (journalistiques), tout à la nouveauté, ne «ronronnent » pas et sont capables de produire sur tel ouvrage de Borges aussi bien un compte rendu fulgurant et visionnaire, qu'une litanie de bêtises - toutes choses également passionnantes à considérer. C'est surtout celle où paraissent en France la plupart des textes marquants concernant l'interprétation de Borges : le Cahier de l'Herne qui lui est consacré en 1964 réunit une bonne partie de ces contributions majeures, le «borgésien " devient une sorte de mot de passe pour happy few, divers «Borges » se constituent: celui d'Étiemble, celui de Blanchot, celui de Foucault, celui de Macherey, celui de Planète, celui de la Nouvelle Critique (on pourrait définir une bonne partie de l'œuvre de Genette - ou, à date plus récente et à d'autres fins, une bonne partie de celle de Pierre Bayard - comme un dialogue avec Borges), celui de la Nouvelle Vague et notamment de Jacques Rivette, qui place son premier film-manifeste, Paris nous appartient (1961), sous l'invocation de Borges, dans un contexte que j'ai commencé à explorer dans ma conférence de clôture au colloque «Borges et la France » de septembre 2009.

14 Je souhaite donc étudier en détail quel est l'auteur, quelle est l'œuvre qui, après être passés par tous ces filtres attendus ou inattendus, obligés ou surprenants (dans un pays comme la France, marqué par une tradition de respect des œuvres traduites), sont donnés aux lecteurs. Viser à une poétique de la réception, c'est faire d'emblée le pari, je le répète, que tous ces filtres, tous ces choix, toutes ces aberrations éventuellement, toutes ces étrangetés en tout cas, produisent des effets puissants, qui se retrouvent dans l'œuvre traduite et qui essaiment dans les lectures qui en sont faites. En d'autres mots: je souhaite montrer la façon dont une réception littéraire translinguistique est construite, sciemment ou non (c'est bien sûr l'étude qui nous dira le degré d'intentionnalité de l'éditeur). D'où l'intérêt, pour moi, de me situer en aval, à la réception, en un lieu où je peux mesurer clairement des traces textuelles ou paratextuelles visibles. Les plus visibles étant constituées par le péritexte éditorial (titres, tables des matières, notices biographiques, préfaces, postfaces, notes...) et par l'épitexte (essentiellement comptes rendus et essais), ainsi que par la façon même dont les textes ont été traduits, toutes ces traces, j'y insiste, ayant pour point commun de marquer un second degré par rapport à la configuration originelle de l'œuvre, de relever d'une recréation qui constitue l'œuvre dans la langue d'arrivée et qui échappe à l'auteur. Par rapport au travail pionnier mené par Sylvia Molloy sur La Diffusion de la littérature hispano-américaine en France au XXe siècle (1972), la principale avancée de ma recherche sera l'attention à ces effets péritextuels, épitextuels et textuels intimes : c'est dire que, à défaut d'une poétique de l'édition, une poétique de la traduction est inséparable de la poétique de la réception que je projette.

Dans mon esprit, il va de soi que la question dépasse largement le cas de Borges, qui présente surtout pour moi l'intérêt de son exemplarité. Se trouve en effet posée la question bien plus générale de la réception de tout écrivain que l'on ne lit pas dans sa langue: dans quelle mesure les choix (ou les aléas) d'édition, de traduction, de diffusion influent-t-ils sur la façon dont il est reçu dans une langue-culture étrangère, comment son œuvre se trouvet-elle modifiée, redessinée, réinterprétée par les filtres supplémentaires qui lui sont 
apposés, voire imposés ? La question n'est pas mineure, puisqu'elle est, au fond, celle de la constitution d'une "littérature universelle» ou d'une "République mondiale des Lettres» au fil des siècles: que reçoit de Kafka ou de Musil un lecteur français non germanophone, que reçoit de Shakespeare ou de Joyce un lecteur français non anglophone ? Un " grand écrivain » résiste-t-il forcément, comme on l'affirme souvent un peu vite, à tout type de «traitement " lié à son passage dans une autre langue, y a-t-il toujours quelque chose de son "génie » qui se sauve, ou au contraire qui se perd (mythe de l'intraduisible) ? Un " grand écrivain » devrait-il être systématiquement "réinventé » par son éditeur, et plus précisément par son traducteur, comme le revendique Alexandre Vialatte à propos de Kafka, parce que ce serait la seule manière de rendre justice à ses inventions (Kafka, inventeur d'un genre et d'une langue, étant d'ailleurs le cas le plus comparable à celui de Borges, au vingtième siècle, de même que le «kafkaïen » est sans doute le seul concurrent du "borgésien ») ? D'où l'intérêt, là encore, de penser en termes de Poétique: c'est une leçon théorique générale que j'ambitionne de tirer d'une telle étude, valant bien au-delà du "cas Borges ", je le répète, ou même du rapport entre langue française et langue espagnole (ou argentine). Mais en même temps, le «cas Borges ", par sa richesse, sa complexité et, sans doute, sa perversité, me donnera la chance d'observer sur la durée des effets de sens (des effets d'écriture et de lecture) nombreux et précis, en tout cas directement liés au second temps d'un transfert linguistique (et culturel). Une fois de plus, je compte sur les aspects excessifs et révolutionnaires de l'œuvre de Borges (qui a donc inventé à la fois un genre nouveau, la "fiction borgésienne ", et une langue nouvelle, un espagnol que nul n'avait jamais écrit avant lui) et sur les effets qu'ils produisent toujours, fût-ce à leur insu et sous d'autres latitudes, pour en tirer des leçons à la fois intimement borgésiennes et, si j'ose dire, universelles.

\section{Écrire, traduire, ménardiser}

16 S'agissant de l'édition, il convient de rassembler les éditions successives de l'œuvre de Borges en français, des origines jusqu'à 1970. En livres, mais aussi en revues, beaucoup de publications pré-originales ayant eu lieu dans des périodiques variés: Confluences, $\mathrm{La}$ Licorne, Les Lettres Nouvelles, Les Cahiers du Sud, Arts, Les Temps Modernes, Tel Quel, Les Nouvelles littéraires, Planète, etc. (la circulation d'une revue à une autre, la façon dont chacune traite cette œuvre étant éminemment significatives - tout comme est significative la relative discrétion avec laquelle $L a N R F$ traite l'Argentin, qui est pourtant un «auteur Gallimard»). Les correspondances (par exemple la correspondance Roger Caillois-Victoria Ocampo), les archives (le fonds Caillois est conservé à Vichy), les journaux intimes (le Borges de Bioy Casares) fourmillent de détails capitaux sur les conditions de cette édition, son contexte, ses accidents.

17 S'agissant de la traduction, le travail consistera à comparer textes de départ et textes d'arrivée, y compris au fil des rééditions successives, tant en espagnol qu'en français (il y a des variations dans les deux langues), pour des parties significatives de l'œuvre: la traductologie, dans sa pratique, n'est pas affaire d'exhaustivité mais d'analyse de séquences et de repérages de figures. Je m'y suis déjà employé, par exemple, dans l'article "Ménard (peut-être sans le vouloir) - Écrire, traduire, ménardiser", pour quelques passages clés de la nouvelle "Pierre Ménard, auteur du Quichotte », en montrant la façon dont le traducteur détruit tous les traits d'humour, comme s'il les trouvait indignes de 
Borges, ou de sa traduction, ou de l'idée qu'il se fait de la littérature. Plusieurs traducteurs étant encore vivants, je les interrogerai sur leur façon de travailler, les éventuelles consignes de l'éditeur, les circonstances de leur choix comme traducteur (plusieurs n'ont traduit qu'un seul livre de Borges), leur intérêt réel pour l'œuvre, etc. Peu d'archives de traducteurs à explorer sans doute, mais par exemple l'hypothèse que Jean Paulhan aurait corrigé de sa main certaines traductions précoces. C'est dire que les archives des éditions Gallimard seront un lieu important de ma recherche sur le terrain.

S'agissant de diffusion, il convient d'obtenir les données des tirages et des ventes, année par année, volume par volume. De retrouver l'appareil éditorial (catalogues, Bulletins de la $N R F$, bandeaux, textes de presse, etc.) accompagnant les lancements et les rééditions.

Enfin, s'agissant de réception, le travail portera sur les articles et les essais spécifiquement consacrés à cette œuvre pendant toute cette période, ainsi que sur les supports les plus divers du "borgésien », déjà évoqués. Ceux-ci incluent les « images » de la personne même de Borges, indépendamment de son œuvre: photographies, informations éparses (la plus spectaculaire dans la période considérée étant l'annonce erronée par une agence de presse française de sa mort !). Une recherche dans les archives des revues et des journaux concernés, ainsi qu'une étude de ces différents types de documents et de leur propre réception, seront évidemment nécessaires.

La France est aussi le pays où naît un "genre mineur " qui fera florès, et où Borges excelle : celui des «entretiens avec Jorge Luis Borges ». Ceux de Jean de Milleret et ceux de Georges Charbonnier paraissent la même année 1967. Publier un livre d'entretiens avec un auteur étranger est assurément un geste fort, en termes de reconnaissance éditoriale et critique; mais seule une analyse approfondie de ces textes généralement négligés nous renseignera sur leurs vrais enjeux.

21 Il est à noter que les revues argentines elles-mêmes, à commencer par la fameuse revue Sur, fondée (en 1931) et dirigée par Victoria Ocampo, réagissent (souvent avec beaucoup de subtilité et d'ironie) à la réception en France de «leur » Borges: une poétique de la réception de l'œuvre de Borges en France doit ainsi passer par une enquête sur le terrain d'origine, à Buenos Aires, autrement dit par une étude de la réception (argentine) de la réception (française). L'interrogation sur l'édition en France ne peut d'ailleurs, plus généralement, faire l'économie d'une interrogation sur l'édition en Argentine : on ne peut exclure que certains "symptômes" éditoriaux français aient été, en quelque sorte, encouragés par la façon dont l'œuvre borgésienne a d'abord été éditée dans son pays d'origine. Les décalages de pays à pays sont toujours éclairants : dans les années 1960, au moment où Borges commence à être reconnu dans son pays comme un immense écrivain (tout en continuant d'être détesté par les péronistes, qui voient en lui l'emblème insupportable de l'oligarchie libérale et du cosmopolitisme anti-argentin), il est lu sur les campus nord-américains comme un auteur hippie ou New Age, et en France même la place privilégiée que lui accorde, dès sa deuxième livraison (1962), la revue Planète, le range sous la bannière " ésotérisme et science-fiction", dont il sera passionnant de voir quels effets de lecture elle a produits, et pour combien de temps...

22 Autre point capital : ce n'est pas seulement la façon dont les revues, les critiques, les intellectuels parlent de Borges qui compte; c'est aussi (n'oublions pas que les années 1960-1970 sont celles du "Boom du roman latino-américain », avec son cortège de « réalisme magique ») ce qu'ils disent sur la littérature latino-américaine en général, le modèle idéal et l'horizon d'attente qu'ils construisent. «Voici le soleil tombant d'aplomb, les prompts orages, les déluges, la mer brûlante, la forêt sans répit », écrit Dominique 
Aury dès 1956 dans une chronique de La NRF consacrée à «La Croix du Sud", où elle pleure sur «l'immense continent [...] qui aurait pu être sauvé de l'homme blanc, et que l'homme blanc, par éclairs de mauvaise conscience, regrette d'avoir arraché aux bisons, aux Indiens nus, aux fabuleux Incas»: cette Amérique fantasmée n'est pas celle de Borges, on s'en doute. Il y a en France, dans les années 1960-1970, dans certains milieux intellectuels et surtout, très majoritairement, dans les milieux universitaires, une haine de Borges, qui est sans doute imputable à la fois à ce « Boom » et aux grands débats politiques du temps, mais aussi, j'en fais l'hypothèse, à la manière de l'éditer. Autrement dit, une poétique de la réception doit inclure une réflexion sur la vision française de la littérature du continent latino-américain, pendant la période considérée, et sur la dimension idéologique de cette vision. Une réception est forcément liée à un contexte (ce qui impliquera un recours à une sociologie de l'édition et de la réception). On ne peut exclure qu'une partie du contexte français se prête idéalement à la diffusion de Borges (sa critique, sa théorisation, son annexion, voire sa fétichisation), tandis qu'une autre partie de ce contexte, parallèlement, la rejetterait.

\section{Éditer Borges}

L'essentiel de ce projet aboutira à la rédaction d'un gros ouvrage, qui pourrait s'intituler (pour des raisons d'efficacité) Traduire Borges. Cet ouvrage suivra sans doute la chronologie notionnelle de la diffusion et de la réception : éditer, traduire, diffuser, lire, critiquer, interpréter (je distingue par ces deux derniers verbes la critique des journalistes et celle des essayistes ou des théoriciens). La partie "traduire ", mais aussi la conclusion générale, devraient être pour moi l'occasion de rassembler les enseignements fondamentaux d'une recherche en traductologie qui m'accompagne donc depuis longtemps, mais que, à la différence de mes autres thématiques scientifiques, je n'ai jamais pu exposer dans un ouvrage de grande ampleur. Je reviendrai notamment sur les deux points qui me semblent les plus novateurs : l'auto-représentation du traducteur (la façon dont le traducteur se met en scène au sein de sa traduction), et la continuité nécessaire, pour le traducteur, entre le texte qu'il traduit et l'œuvre. Quand on traduit un texte ou un livre, de fait, on ne traduit pas seulement un texte ni un livre, on traduit une œuvre, on traduit un auteur, et on traduit même la bibliothèque d'un auteur, je veux dire l'ensemble des livres qu'il a écrits avant (et après) celui que l'on traduit, voire l'ensemble de ceux qu'il a lus et qu'il réécrit. Si l'on n'a pas lu tout Borges, si l'on ne connaît pas les principales sources (latines, anglaises, françaises, italiennes, allemandes...) de son inspiration, on ne peut savoir que telle séquence textuelle est reconduite inexorablement sous sa plume depuis les années 1930 jusqu'aux années 1980, et l'on risque donc de la sous-traduire. À quoi s'ajoute que le texte borgésien joue parfois à anticiper et à brouiller la façon dont il sera traduit, par une série de processus narratifs retors que j'ai étudiés dans l'essai où je propose la notion de « texte traducteur $»^{1}$.

La question de l'édition est une de celles qui se posent avec le plus d'acuité, aujourd'hui, aux spécialistes des littératures étrangères, avant tout parce qu'elle interroge leur place dans la société où ils vivent, en pointant une de leurs fonctions sociales irremplaçables : procurer aux lecteurs de leur pays des corpus littéraires capitaux qui sans eux resteraient ignorés, incomplets ou faussés. Depuis vingt-cinq ans (Borges est mort en 1986), beaucoup de choses se sont passées dans le domaine de l'édition borgésienne. Des textes inédits ou rares ont été édités de manière systématique. Des bases de données capitales ont été 
mises en ligne (l'Argentin Nicolás Helft, directeur du plus grand fonds d'archives borgésiennes au monde, a mis en ligne à la fois la totalité des numéros de la revue Sur, à laquelle Borges a donné un nombre considérable de publications pré-originales, et une base sur la vie et l'œuvre de Borges), il a également publié la première Bibliographie complète de ce corpus. D'autres livres importants sont parus, certains sous ma plume (édition des Cours de littérature anglaise en 2006, au Seuil, puis de deux manuscrits de fiction en fac-similés en 2010, aux Presses Universitaires de France, en coédition avec la Fondation Martin Bodmer de Genève, où sont conservés ces manuscrits). La Bibliothèque Nationale de Buenos Aires vient de se procurer une magnifique édition des marginalia inscrites par Borges sur des livres de sa bibliothèque donnés à l'institution dont il fut le directeur. Une toute première "édition critique» de l'œuvre borgésienne paraît en plusieurs volumes à Buenos Aires, longtemps après l'édition française de la Pléiade: l'Argentine est capable de réussites éditoriales prodigieuses (l'Histoire critique de la littérature argentine en douze volumes, dirigée par Noé Jitrik, ou, à une échelle plus modeste, la publication de la correspondance de Manuel Puig, des œuvres complètes d'Osvaldo Lamborghini ou des inédits de Silvina Ocampo), mais elle n'avait quasiment aucune notion, à date récente, de ce qu'est une édition critique ou génétique (mon édition des « romans complets » de Bioy Casares dans la collection « Bouquins », par exemple, n'a pas d'équivalent en espagnol). Si l'on sort du domaine borgésien, les Archives de l'Unesco ont fourni des corpus exemplaires (éditions génétiques et critiques) des littératures d'Amérique centrale et d'Amérique du Sud, d'autres initiatives remarquables prenant maintenant le relais, en Amérique latine et en France - où, sous l'impulsion des projets conduits au CNRS par l'Institut des textes \& manuscrits modernes (ITEM), ce sont évidemment les corpus littéraires français qui occupent le devant de la scène. L'apparition de l'édition numérique en ligne (en pointe à Grenoble, où sont conservés plusieurs dizaines de milliers de folios de journaux de Stendhal) pose la question du sens qu'il y a à continuer à produire des éditions sur papier, a fortiori dans le cas d'un Borges, qui est par essence un hyper-écrivain et dont certains font même un précurseur de l'hypertexte et du Web. Voilà pourquoi il me semble important d'organiser une réflexion collective sur l'état actuel de l'édition de Borges dans le monde (sans refuser les exemples connexes), de répertorier et de comparer les options suivies, pour en arriver à une série de propositions d'action: puisque beaucoup de choses restent à faire dans ce domaine, pourquoi ne pas essayer de les mener à bien en tirant les conséquences du passé, ou pourquoi, au moins, ne pas émettre un certain nombre de recommandations à l'intention des éditeurs, voire des (re)traducteurs, à venir. Il n'est pas exclu que j'édite à moyen ou long terme les manuscrits d'essais également conservés à la Fondation Martin Bodmer de Genève, si possible en mettant en place et en pilotant une équipe d'éditeurs.

\section{Coda}

Si je reviens sur mes principales thématiques de recherche depuis trente ans, elles sont, je crois, marquées par l'originalité de leurs objets successifs, mais aussi par leur continuité souterraine, comme un feuilletage d'enjeux théoriques successifs qui ne cesseraient de se recouvrir et de s'enrichir. Elles sont aussi caractérisées par des allers-retours constants entre le corpus fondateur qu'a été pour moi celui de Borges, et beaucoup d'autres littératures (à commencer par la littérature anglaise), dont je suis devenu spécialiste par « devoir borgésianiste ». La réécriture dans l'œuvre de Borges (la mise en rapport de ses 
textes avec des textes antérieurs de lui ou d'autres auteurs, la mise en évidence de la façon dont la fiction lui permet de remodeler ses "autobiographèmes" et de se construire un mythe d'auteur, tout en s'en nourrissant, la mise au jour au sein de son œuvre de véritables réseaux de textes où une même situation narrative se trouve exploitée de toutes les façons possibles, jusqu'à épuisement) était une manière, comme je l'ai déjà dit, sous l'invocation de l'essai fondateur de Genette, «La littérature selon Borges » (1964), d'opter précocement pour la pluralité textuelle, dont les jeux et les enjeux m'ont toujours semblé encore plus subtils et vertigineux que ceux que peut déployer un texte unique, fût-il borgésien.

Les recherches que j'ai menées sur les formes brèves (nouvelle, "nouvelle exemplaire » de Cervantès, cuento, conte, « fiction » de Borges, novelita de César Aira, préface, énigme, histoire drôle, fable, essai) ou hyper-brèves (le micro-conte de science-fiction: voir la dizaine de numéros de revues que j'ai dirigés sur ces formes entre 1988 et 2003) ont été également une façon de dépasser le texte en soi et de penser la dualité, la complémentarité, la compétition, la contradiction : penser la forme brève contre la forme longue «impérialiste » qu'est le roman; la forme brève contre la forme fixe; la forme brève contre la forme inclusive (mon essai sur la mise en recueil des nouvelles est, avec celui sur la poétique de la forme brève et celui sur la préface de Borges à L'Invention de Morel de Bioy Casares, un de ceux auxquels je tiens le plus).

Mes longues années de pratique et de théorisation de la traductologie interrogent la "boîte noire» d'une opération que les traductologues sautent souvent un peu rapidement. Mes recherches réunissent traducteur et auteur sous l'angle que j'ai déjà évoqué, comme "coauteurs» de deux textes (deux «brouillons", dirait Borges, sur la chaîne interminable des réécritures) qui sont à la fois le même texte et deux textes différents.

Les quinze années que j'ai consacrées, parallèlement au reste de ma vie scientifique, à l'écriture en collaboration, en grande partie grâce à ma première délégation à l'IUF (la recherche a démarré en 1990, pour se matérialiser par un premier ouvrage en 2006 : Nous est un autre - Enquête sur les duos d'écrivains, en collaboration avec Benoît Peeters; une seconde partie, plus strictement théorique, est à venir) sont comme un prolongement géométrique de ma recherche en traductologie : non plus deux auteurs pour deux textes, mais deux auteurs pour un seul texte, comme un défi au dogme de l'auteur unique, dans les domaines les plus variés: littératures française, italienne, anglaise, allemande, espagnole et argentine, roman populaire, roman policier, théâtre, poésie, journal intime, récit de voyage, philosophie, psychanalyse, cinéma, bande dessinée.

Le séminaire que je conduis depuis trois ans sur la notion de romanesque m'aide à penser aussi bien les apories narratives borgésiennes (la manière dont une fiction de dix pages peut rivaliser, en complexité, en vertiges et finalement en longueur avec l'épaisseur d'un roman de mille pages) que le lien un peu mystérieux entre un texte et ses brouillons, les variantes préparatoires racontant à leur manière le roman de la fiction (je renvoie à mon édition récente de deux manuscrits borgésiens).

Enfin, la recherche maintenant programmée, comme l'interrogation d'un nouvel Autre, qui une fois encore constitue un double de l'auteur : la figure de l'éditeur (au sens le plus large, comme on l'aura compris, puisque étendue à la chaîne éditeur-traducteur-critique). Tout converge, d'une certaine manière, dans ce nouveau projet: la «transcendance 
textuelles des textes ", la traductologie, la question des formes et des genres littéraires, la collaboration et même, on l'a vu, le romanesque.

31 Si j'ai un talent comme poéticien, c'est peut-être celui de regarder là où les autres ne regardent pas. Le livre sur l'écriture en collaboration, que nous avons donc écrit en collaboration avec Benoît Peeters, est le premier au monde à tenter une analyse systématique d'un phénomène pourtant ancien et fréquent, mais que personne n'avait jamais considéré. La continuité heureuse et opératoire (puisqu'elle ne cesse de me pousser à avancer) qui s'est tissée pour moi entre ces objets successifs ou simultanés de recherche est faite des symétries, des échos, des contradictions, des paradoxes que je viens d'évoquer, mais elle est sans doute aussi, au plus profond, faite de cette curiosité pour des phénomènes invisibles, qui sont comme autant de tabous de la Poétique. C'est cette curiosité que je souhaiterais appliquer, dans les années qui viennent, à ce nouvel objet qu'est la « rencontre » de l'auteur étranger et de son éditeur, via le traducteur et sous le regard ultime du lecteur. Ce serait pour moi l'occasion de boucler de la plus belle des façons l'ensemble de mes recherches borgésiennes et, indissociablement, poéticiennes.

\section{NOTES}

1. Deux autres livres sont prévus pour cette période, La Pierre de Lune (Une humble apologie du romanesque) et le Journal de voyage de Bioy Casares chez Christian Bourgois ; ainsi que l'organisation de deux colloques internationaux sur Borges et d'un séminaire international itinérant.

\section{RÉSUMÉS}

Michel Lafon a décidé en 2011 de présenter sa candidature à l'Institut universitaire de France (IUF) alors que son activité d'écrivain et de scénariste avait pris une place considérable dans sa vie, pour prolonger et réinventer son infatigable dévouement à Jorge Luis Borges.

Nous reproduisons ici le descriptif du projet, dont les premiers paragraphes dévoilent des biographèmes du futur « borgiste », qui montre à quel point, alors qu'il est l'auteur d'une œuvre incontournable et édifiante sur l'auteur argentin, tout semble à faire. Michel Lafon avait, il le dit lui-même, un talent comme poéticien, « celui de regarder là où les autres ne regardent pas ».

Michel Lafon decided in 2011 to run for the IUF even so when his activity as a writer and screenwriter had taken an important place in his life, to extend and reinvent his tireless dedication to Jorge Luis Borges.

We reproduce here the project, which first paragraphs unveil biographemes of the future "borgiste", that shows how, while he is the author of an essential work on the Argentine writer, 
that everything seems to be done. Michel Lafon had, as he told himself, a talent like poetician, "the one to look where others are not looking."

Michel Lafon decidió en 2011 presentar un proyecto de investigación en el prestigioso Instituto universitario de Francia aún así cuando su actividad como escritor y guionista ocupaban un lugar cada vez más importante en su vida, prolongando y reinventando su incansable dedicación a Jorge Luis Borges.

Reproducimos aquí el descriptivo del proyecto, cuyos primeros párrafos revelan algunos biografemas del futuro "borgista", que muestra hasta qué punto, ahí cuando es autor de una obra insoslayable y decisiva sobre el autor argentino, parece que todo queda por hacer. Michel Lafon tenía, como él mismo lo sugiere, un talento como teórico de una poética, « el de mirar ahí donde nadie mira ».

\section{INDEX}

Palabras claves : editar a Borges, recepción de Borges en Francia, traducciones de Borges

Keywords : editing Borges, Borges reception in France, Borges translations

Mots-clés : éditer Borges, réception de Borges en France, traductions de Borges 